\title{
Customized Oral Hygiene Therapy A Personalized Approach
}

\author{
Vaishnavi Bhaskar', Martha McComas², Chin Wei (Jeff) Wang ${ }^{3 *}$ \\ ${ }^{1} B D S$ MPH DDS candidate, University of Michigan School of Dentistry \\ ${ }^{2}$ RDH, MS, Clinical Assistant Professor, Department Of Periodontics and Oral Medicine, Division of Dental Hygiene \\ ${ }^{3}$ DDS, DMSc, Clinical Assistant Professor, Department of Periodontics and Oral Medicine, University of Michigan School of Dentistry
}

\begin{abstract}
Received: November 11, 2016; Accepted: November 26, 2016; Published: December 09, 2016
*Corresponding author: Dr. Chin Wei (Jeff) Wang, DDS, DMSc, Clinical Assistant Professor, Department of Periodontics and Oral Medicine, University of Michigan School of Dentistry, 1011 N. University Ave., Ann Arbor, MI 48109-1078, Tel: 734-647-6175; E-mail: jeffwa@umich.edu
\end{abstract}

\begin{abstract}
Oral hygiene instruction is a challenging, yet critical therapy. Successful and stable outcomes of Oral Hygiene Therapy (OHT) are often hindered by patient's motivation, anatomical and technical barriers.Everyindividual presentsa diverseset of challenges thataffect their motivation and oral hygiene practices. Thus, personalized and tailored instructions are required for each individual. The following case report demonstrates the dynamic changes that can occur when implementing a customized OHT using a unique combination of nontraditional and traditional oral hygiene instructions and the use of a new plaque control heat map. The basic elements of OHT used in this case included Motivational Interviewing, interactive demonstrations of evidence-based targeted approach with specific oral hygiene aids, and behavior change monitoring program. It was observed that this personalized approach rendered clinically significant improvements on patient's oral hygiene and general well-being.
\end{abstract}

Keywords: Oral Hygiene Therapy; Customized; Patient Education; Oral Health;

\section{Abbreviations}

BOP: Bleeding on Probing; OHT: Oral Hygiene Therapy; SRP: Scaling and Root Planning; PI: Plaque Index; PCR: Plaque Control Record; MI: Motivational Interviewing; ULQ: Upper Left Quadrant; LLQ- Lower Left Quadrant; URQ: Upper Right Quadrant; LRQ- Lower Right Quadrant

\section{Introduction}

In September of 2016 the World Dental Federation (FDI) published an executive summary defining "oral health". [1] This group defined oral health as "multi-faceted and includes the ability to speak, smile, smell, taste, touch, chew, swallow and convey a range of emotions through facial expressions with confidence and without pain, discomfort and disease of the craniofacial complex". [1] To accomplish this, practitioners must develop and utilize effective "chair-side" models of oral hygiene instructions so patients can develop the skills needed to maintain their own oral health. [2] The past highly paternalistic model of "practitioner talk patient listen" has proven extremely ineffective when encouraging patients to adopt behavior change. [3]
Dental professionals are aware that the overall success of periodontal therapy is directly related a patient's daily oral hygiene. Therefore, the OHT offered to patients should incorporate principles from behavioral science, such as motivational interviewing. [4] It is suggested that for patients to adopt new skills they must be involved in the learning, i.e. Chairside active demonstrations. [5] Currently MI is one strategy used in hopes of encouraging lasting behavior change. Motivational interviewing can identify barriers to change, allowing the practitioner to tailor their educational approach. This strategy has shown effective in leading patients to adopt the new changes or strategies suggested by their health care provider. [2, 4] Therefore, combining the spirit of MI and the traditional social learning theory [6] technique of "active demonstration" dental health care practitioners can individually customize their oral hygiene instructions given during OHT sessions.

The following case report explores the use of Customized Oral Hygiene Therapy (COHT), a delivery method that combines the spirit of MI, active demonstration, as well as behavior change monitoring.

\section{Case Presentation And Treatment Planning}

A healthy 25- year old male patient reported to the clinic for a comprehensive exam and prophylaxis. His diagnosis was localized moderate chronic periodontitis. In addition, clinical examination revealed poor oral hygiene, significant plaque and calculus deposits, developmental defects of the enamel and severe crowding and malocclusion. At the initial appointment a comprehensive overview of his baseline dental literacy and perceived value of dental care was obtained. The proposed initial comprehensive periodontal treatment plan included four quadrants of scaling and roots planning (SRP) with customized oral hygiene therapy and a 12-week re-evaluation.

\section{Methods}

To gather data we used a plaque control heat map created by the corresponding author. This index combines both a visual and a numerical index of plaque accumulation; a combination of the plaque index (PI) (Stillness and Loë) $[7,8]$ and the plaque 
control record (PCR) (O'Leary) [9] to capture both spatial and quantitative patterns.

Disclosing solution was used to highlight the areas and quantity of plaque retention, where a blunt probe used to quantify the thickness of plaque accumulation. Areas with both high quantity and gross thickness were recorded and then termed "hot spots." This was then converted into a grey scale where scores of 3 are indicated with black, 2 with dark gray, 1 with light gray and 0 with white. In addition, intraoral photos were also evaluated (Figures 2-4). Once the "hot spots" were recorded and tabulated, they were analyzed from which customized oral hygiene instructions were developed. Evidencebased recommendations and oral aids were selected to improve his oral hygiene while simultaneously appealing to his social determinants for health. From the heat map analysis the use of new oral aids and strategies were suggested and demonstrated for use only in the quadrants where the treatment had been completed. This quasi-experimental design was used so that the clinician and the patient can see the impact of both clinical treatment and behavior change.

Initially, oral hygiene recommendations were developed based on local risk factors; anatomical entities such as crowding lingual positioned teeth, and the presence of developmental enamel defects. (Figure 1) During the second visit, the patient was asked to demonstrate his "newly acquired" brushing technique. At this time the presence of plaque that remained on the inter-proximal aspect of teeth despite thorough brushing was highlighted. Guided by the heat map, a floss pick was introduced through active demonstration so the patient could see the stained plaque dissipate. Each subsequent visit included COHT using the spirit of MI and active demonstration techniques. Once the disclosing solution was applied, the patient was able to visualize the "hot spots", areas he would need to focus on at home. (Figures 5-6). The practitioner then demonstrated how to use evidence-based adjunctive oral aids in the patient's mouth while watching through a hand mirror. Finally, the patient was asked to repeat the techniques under practitioner guidance for added reinforcement. During the MI portion of the intervention it was discovered that the patient found motivation for behavior change through science. Therefore, in hopes of piquing his personal motivation, a paper outlining the scientific evidence regarding the correlation between periodontal disease and cardiovascular

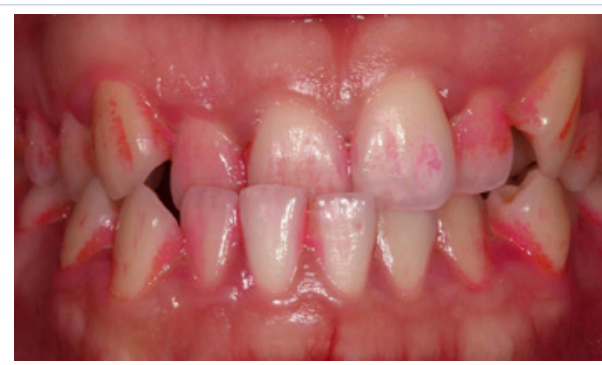

Figure 1:Initial presentation with poor oral hygiene revealed with disclosing agent. Generalized plaque accumulation, especially interproximal and embrasure surfaces.

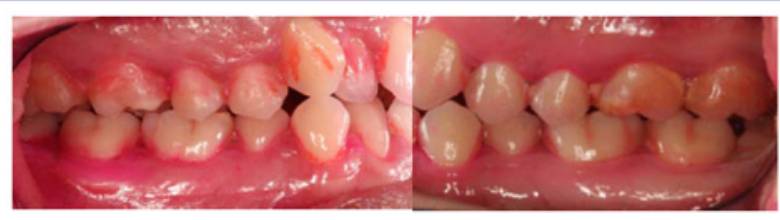

Figure 2: The proximal surface and embrasure space, especially between \#18 and \#17 (horizontally impacted wisdom tooth as a local factor) is a "hot spot" for plaque accumulation.

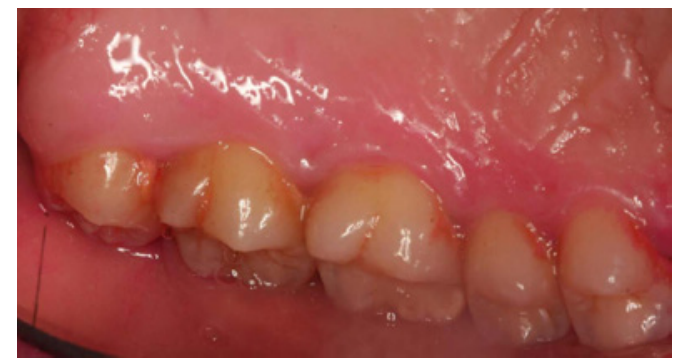

Figure 3: Significant plaque accumulation on the palatal cervical and embrasure aspect demonstrating the patient's lack of awareness about paying attention to brushing all tooth surfaces.

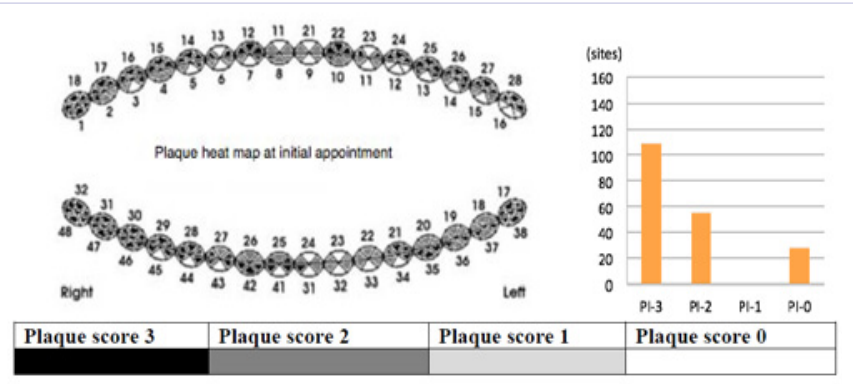

Figure 4: Plaque control heat map demonstrates generalized heavy plaque accumulation (Score 3 ) especially around the posterior teeth.
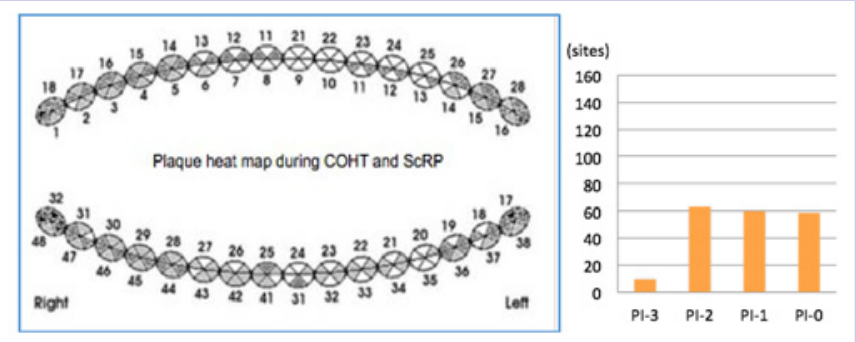

Figure 5: A definite reduction in the plaque score is observed, the patient also reported a reduction in bleeding from his gums while brushing. Plaque scores of 3 is observed on the third molars and the importance of having these teeth extracted was further reinforced.

disease was shared, and earth friendly products were introduced. [10]

\section{Results}

Significant improvement in oral hygiene could be seen both visually and through scoring on the heat map. (Figures 


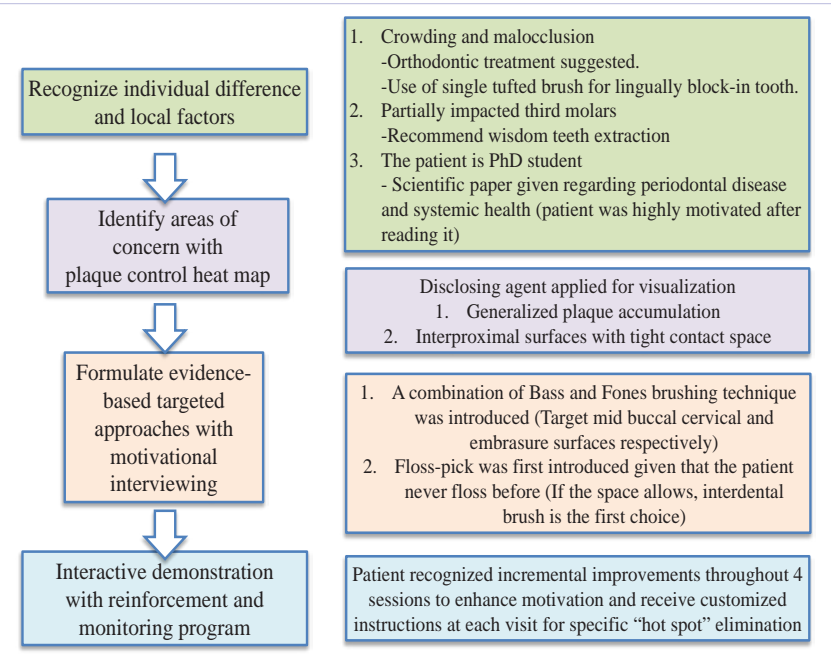

Table 1: Algorithm for developing COHT for this patient-
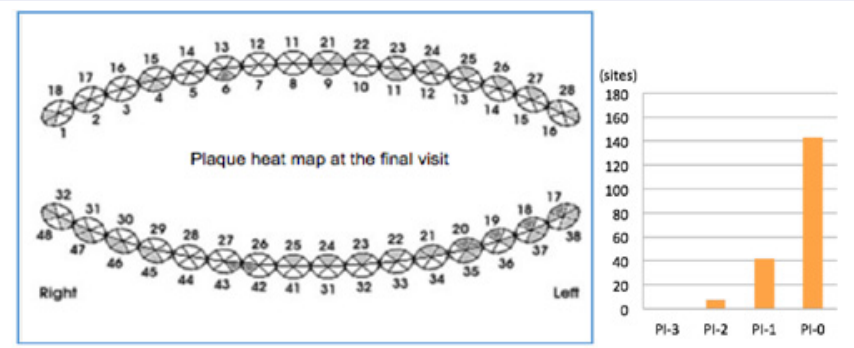

Figure 6: Substantial reduction in plaque scores was observed at the end of SRP. Disclosing solution was applied and only thin flecks of plaque was noted at the embrasure areas on the third molars.

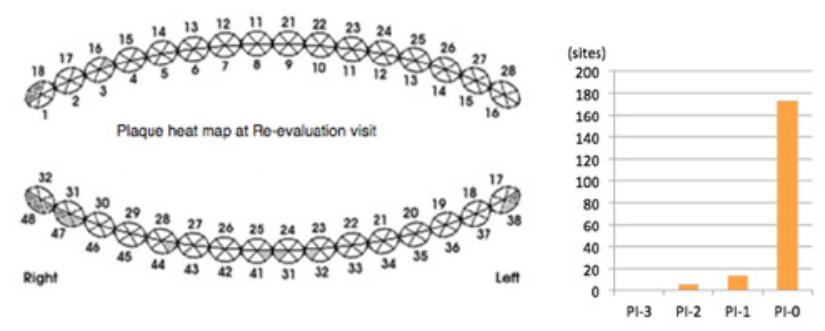

Figure 7: Presence of very minimal (if any) inter proximal plaque was seen. The only hot spot that remained was on the distal aspect of \#31 (especially around the impacted tooth.

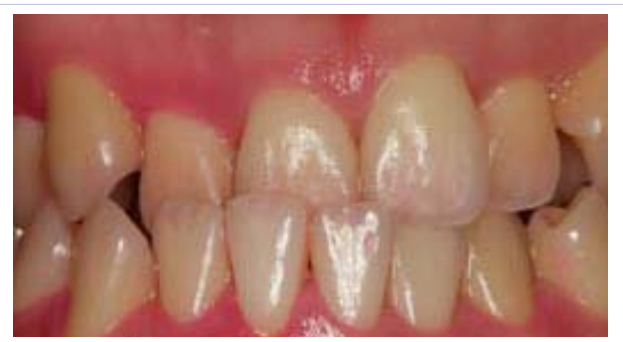

Figure 8: Intra oral photographs after the application of disclosing solution taken 12 weeks after SRP and COHT showed significant improved oral hygiene

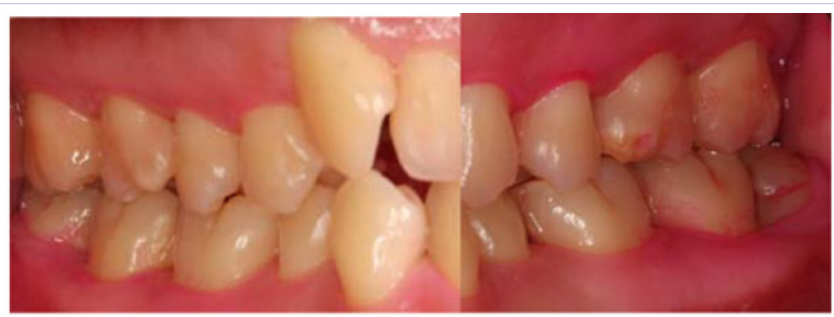

Figure 9: Intra oral photographs after the application of disclosing solution taken 12 weeks after SRP and COHT-generalized improvement but residual plaque associated with the third molars observed

$6-7 ; 8-10)$. At the initial consultation $56.7 \%$ of the surfaces had scored a plaque score of 3 . This overall plaque score decreased to $0 \%$ by the conclusion of the treatment plan. In addition, the patient was able to sustain his oral hygiene between the final session and the re-evaluation appointment scheduled 12 weeks later. (Figure 6 \&7) It was evident that the daily oral hygiene care significantly impacted the health of his periodontium which was demonstrated by the dramatic decrease in the number of points of bleeding on probing.

Most importantly, upon treatment completion the patient stated that "the oral hygiene therapy sessions and his newly improved oral hygiene were positively impacting his general well-being." He also reported that "he felt more comfortable in social situations and felt his confidence and self-esteem had improved." It is also important to note that he stated he "felt an overall improvement in his appearance, mood and happiness."

\section{Discussion}

Oftentimes practitioners attempt to change a patient's behavior through a prescriptive approach which has been found to be quite ineffective in promoting lifelong behavior changes. $[2,4]$ Incorporating behavior science through the use of personal counseling and motivational interviewing has shown effective in empowering lasting behavior changes. $[2,4]$ Our COHT is a blended approach to oral hygiene instruction that uses a combination of MI and traditional education delivery methods. Parallel to the literature, we can say with confidence that this combination improved patient compliance through eliciting behavior change. [4] The literature suggests that by involving the patient in their decision to change will increase the patient's self-efficacy and perceived benefits of the behavior change, thus creating overall improved and lasting oral health. [7 ] Using the heat map as both a qualitative and quantitative behavior change record falls in line with literature that suggesting that goal setting and continuous behavior change monitoring also encourage a lifetime of change. [7] Empowering the patient to understand the cause of the disease process and encouraging him to follow the outcomes enabled him to make a high commitment for a positive behavior change.

In this case, there were clinically evident signs of oral health improvement, both in terms of daily oral hygiene and in terms of gingival inflammation. These findings demonstrate the success 
of using such targeted and customized approach to OHT. The outcomes of this case validated the relevance of using MI as a way of appealing to the patient's wide background of social, economic, cultural, and environmental situations, while continuing to demonstrate, guide, and monitor outcomes is a successful way of eliciting lasting behavior change.

\section{Acknowledgements}

This study is supported by Gilbert Whitaker Grant from the University of Michigan Center for Research on Learning and Teaching (CRLT)

\section{References}

1. Glick M, Williams D, FDI Think Tank. A new definition for oral health An executave summary.[Internet]. World Dental Federation; 2016 October 16 [Updated 2016 October 13; cited 2016 November 10] .

2. Yevlahova D, Satur J. Models for individual oral health promotion and their effectiveness: a systematic review. Aust Dent J. 2009;54(3):190197. doi: $10.1111 / \mathrm{j} .1834-7819.2009 .01118 . x$.
3. Satur J, Gussy M, Morgan M, Calache H, Wright FA. Evidence based review of oral health promotion. Consortium of the University of Melbourne Co-operative Research Centre for Oral Health Science and Dental Health Services Victoria. 2006.

4. Wilder RS, Bray KS. Improving periodontal outcomes: merging clinical and behavioral science. Periodontology 2000. 2016;71(1):65-81. doi: $10.1111 /$ prd.12125.

5. Löe H. Oral hygiene in the prevention of caries and periodontal disease. International dental journal. 2000;50(3):129-139.

6. Newton JT, Asimakopoulou K. Managing oral hygiene as a risk factor for periodontal disease: a systematic review of psychological approaches to behaviour change for improved plaque control in periodontal management. Journal of clinical periodontology. 2015;42(S16):36-46. DOI: $10.1111 /$ jcpe.12356.

7. SilnessJ,LöeH.Periodontal disease in pregnancy II.Correlation between oral hygiene and periodontal condition. Actaodontologicascandinavica. 1964;22(1):121-135. doi.org/10.3109/00016356408993968.

8. Löe H. The gingival index, the plaque index and the retention index systems. Journal of periodontology. 1967; 38(6):610-616. DOI: 10.1902/jop.1967.38.6.610. 